\title{
Rational Quadratic Approximation to Real Plane Algebraic Curves ${ }^{1)}$
}

\author{
Xiao-Shan Gao and Ming Li \\ Key Lab of Mathematics Mechanization \\ Institute of Systems Science, AMSS, Academia Sinica \\ Beijing 100080, China \\ (xgao,mli)@mmrc.iss.ac.cn
}

\begin{abstract}
An algorithm is proposed to give a global approximation to an implicit real plane algebraic curve with rational quadratic B-splines. The algorithm consists of three steps: curve segmentation, segment approximation and curve tracing. The curve is first divided into so-called triangle convex segments. Then each segment is approximated with several rational quadratic Bézier curves. At last, the curve segments are connected into several maximal branches and each branch is represented by a Bspline curve resulting in a $C^{1}$ global parameterization for the curve branch. Due to the detailed geometric analysis, high accuracy of approximation may be achieved with a small number of quadratic segments. The final approximation based on quadratic spline curves keeps many important geometry features and gives a refined topological structure of the original curve. Extension of the method to spatial curves is also illustrated.
\end{abstract}

Keywords: plane algebraic curve, parametrization, approximation, quadratic Bézier curve, quadratic B-spline curve, topology determination.

\section{Introduction}

An implicit real plane algebraic curve of degree $n$ is defined by $f(x, y)=0$ where $f(x, y)$ is a polynomial of degree $n$. The curve is said to be rational if it can be additionally represented by a rational parametric equation $x=\frac{x(t)}{d(t)}$ and $y=\frac{y(t)}{d(t)}$, where $x(t), y(t), d(t) \in \mathcal{R}[t]$ are of degrees at most $n$. Implicit and parametric representations of algebraic curves have important applications in CAGD [18].

It is desirable to generate the parametric representation for an implicitly given algebraic curve, because with the parametric representation we can easily compute points on the curve and plot it. Unfortunately, only a small subset of real algebraic curves are rational. In general, an algebraic curve of arbitrary degree is rational if and only if its genus is zero [21]. Methods to find parametric equations of implicit curves are given, for instance, in $[1,8,19,20]$. For general implicit curves, approximation methods are therefore put forward to give a rational form. These methods might be categorized mainly into three classes: the linear approximation [13], the points sampling approximation [16, 17, 22] and the approximation based on power series $[2,6]$. Ihm surveyed a number of techniques for generating a piecewise

Partially supported by a National Key Basic Research Project of China (NO. G1998030600) and by a US NSF grant CCR-0201253. 
linear approximation for an algebraic curve [13]. Given a model shape, curve or surface, expressed by a set of sample points on it, Pottmann used an active B-spline curve or surface to approximate it with an optimization method [16]. The active B-spline curve method is further refined in [22]. For a polygonal curve in space, whose vertices can be generated through adaptive sampling, Safonova and Rossignac gave a compressed piecewise-circular approximation for it [17]. Chuang and Hoffmann gave a local approximation to a plane curve with power series [6]. Bajaj and Xu used a combination of algebraic and numerical techniques to construct a $C^{1}$-continuous, piecewise rational approximation of a plane algebraic curve [2]. There the Hensel lifting technique and Padé approximation are used.

As an extension of our previous work on approximate implicitization [10], we consider in this paper the approximate rational parametrization problem for a plane algebraic curve $\mathcal{C}$ within a bounding box $B$ (written as $\mathcal{C}_{B}$ for short) from a geometrical viewpoint. The resulted approximations are several rational quadratic B-spline curves, each of which is obtained from piecewise rational quadratic Bézier segments. The rational quadratic segment is used since it is the freeform curve with the lowest degree and has many nice properties [7, 14]. The algorithm consists of three steps: curve segmentation, segment approximation and curve tracing. First, the curve is divided into triangle convex segments (definition in section 2.1.). Then each triangle convex segment is approximated with several rational quadratic Bézier curves. At last, the curve segments are connected into several maximal branches and each branch is represented globally by a B-spline curve resulting in a $C^{1}$ representation for the curve branch.

As compared with previous approaches, our method is based on a detailed topology analysis of $\mathcal{C}_{B}$. Previous methods such as [2] also assumed that the curve topology was known. In this paper, after a topology is determined with method in [11, 12], we further try to find the inflection points and tangent directions of the curve so that triangle convex segments are obtained. The triangle convexity of the segment make it possible to give a nice quadratic approximation with a method called shoulder point approximation. Due to these facts, high accuracy of approximation may be achieved with a small number of quadratic segments. The final approximation based on quadratic spline curves keeps many important geometry features of the original curve such as the convexity and sharp points. The branches obtained in the tracing step provide a global parameterization and a refined topological structure of the curve.

The rest of the paper is organized as follows. The curve segmentation, the segment approximation and the curve tracing are illustrated in sections 2., 3. and 4. respectively. After the main algorithm and some examples are given in section 5., we conclude this paper in section 7 ..

\section{Curve segmentation}

Throughout this paper, we assume that $f(x, y) \in Z[x, y]$ is an irreducible polynomial of degree greater than two. A plane algebraic curve $\mathcal{C}$ is defined by $f(x, y)=0$ and $\mathcal{C}_{B}$ is taken for the part of $\mathcal{C}$ inside a bounding box $B=\left\{(x, y): x_{l} \leq x \leq x_{r}, y_{b} \leq y \leq y_{u}\right\}$. And the approximation error bound is assumed to be a small positive number $\delta$.

\subsection{Preliminaries about plane algebraic curves}


A point $P=\left(x_{0}, y_{0}\right)$ is said to be a singular point of $\mathcal{C}$ if $f\left(x_{0}, y_{0}\right)=f_{x}\left(x_{0}, y_{0}\right)=$ $f_{y}\left(x_{0}, y_{0}\right)=0 . \quad P$ is said to be a vertical point of $\mathcal{C}$ if it is a non-singular point and $f\left(x_{0}, y_{0}\right)=f_{y}\left(x_{0}, y_{0}\right)=0$. The inflection points, flexes for short, of $\mathcal{C}$ are its non-singular points satisfying the Hession equation $H(f)=0$ [21].

A curve segment $S$ of $\mathcal{C}$ is an open ended and continuous part of $\mathcal{C}$ with two endpoints $P_{0}$ and $P_{1}$. The left (right) endpoint is the one with smaller (larger) $x$ coordinate. If $P_{0}$ and $P_{1}$ have the same $x$ coordinate, then the left (right) endpoint is the one with smaller (larger) $y$ coordinate.

Let $P_{0}$ be an endpoint of a curve segment $S$. Then a tangent direction $T_{0}=\left(x_{t}, y_{t}\right)$ of $S$ at $P_{0}$ always exists [21]. If $P_{0}$ is the left endpoint, $T_{0}$ is called the left tangent direction of $S$, and is denoted by $T_{-}$. The left slope $k_{-}$of $S$ at $P_{0}$ is defined as follows: if $x_{t} \neq 0$, $k_{-}=y_{t} / x_{t}$; otherwise $k_{-}=+\infty$ or $-\infty$ depending on $y_{t}>0$ or $y_{t}<0$. The right slope $k_{+}$ and the right tangent direction $T_{+}$can be defined in a similar way.

We use $S\left[P_{0}, P_{2}\right]$ to denote a curve segment of curve $\mathcal{C}$ with left endpoint $P_{0}$ and right endpoint $P_{2}$ and $S\left[P_{0}, T_{0}, P_{2}, T_{2}\right]$ is also used when the left and right tangent directions $T_{0}$ and $T_{2}$ are also prescribed.

A curve segment $S\left[P_{0}, T_{0}, P_{2}, T_{2}\right]$ is said to be triangle convex if either

1. the lines passing through $P_{0}$ and $P_{2}$ and with directions $T_{0}$ and $T_{2}$ respectively meet at a point $P_{1}$ and the line segment $P_{0} P_{2}$ and $S$ form a convex region inside the control triangle $P_{0} P_{1} P_{2}$ of $S$; or

2. $T_{0}$ and $T_{2}$ are parallel and the line segment $P_{0} P_{2}$ and the curve segment $S$ form a convex region.

$S_{P}$ is said to be a shoulder point on a triangle convex segment $S\left[P_{0}, P_{2}\right]$ if $S_{P}$ has the maximal distance to the line $P_{0} P_{2}$.

Lemma 2.1 The shoulder point for a triangle convex segment $S\left[P_{0}, P_{2}\right]$ is unique.

Proof. Suppose that there are two shoulder points $S_{1}$ and $S_{2}$. Since $S\left[P_{0}, P_{2}\right]$ is triangle convex, the line segment $S_{1} S_{2}$ should lies inside the region formed by line $P_{0} P_{2}$ and $S$. Since $S_{1}$ and $S_{2}$ have maximal distance to $P_{0} P_{2}$, the line segment $S_{1} S_{2}$ must be coincident with $S$. This is impossible because $f(x, y)$ is irreducible and of degree greater than two.

From a set of curve segments $\mathcal{S}_{S}$, we can generate a plane graph $\mathcal{G}_{S}$ with a map $\mathcal{U}: \mathcal{S}_{S} \rightarrow$ $\mathcal{G}_{S}$ such that

1. $\mathcal{G}_{S}$ takes the set of the endpoints of the segments in $S_{S}$ as its vertex set $V\left(\mathcal{G}_{S}\right)$, and

2. there exists an edge between two vertices $v_{1}, v_{2}$ if and only if $v_{1}, v_{2}$ are the endpoints of a segment in $\mathcal{S}_{S}$. We use $E\left(\mathcal{G}_{S}\right)$ to denote the set of edges in $\mathcal{G}_{S}$.

It can be seen that $\mathcal{U}$ is a bijection map and $\mathcal{U}^{-1}$ is used to denote the reverse.

The degree of a vertex $v$ in $\mathcal{G}_{S}$ is the number of edges in $\mathcal{G}_{S}$ incident with $v$.

\subsection{Topology determination}

The topology determination of $\mathcal{C}_{B}$ produces a plane graph $\mathcal{G}_{T}$ which is topologically equivalent to $\mathcal{C}_{B}[11,12]$. This is the starting point of our algorithm. Roughly speaking, the algorithm works as follows. 
Algorithm 2.2 (Topology Determination[11, 12]) The input is a plane algebraic curve $\mathcal{C}_{B}$. The output is a plane graph $\mathcal{G}_{T}$ topologically equivalent to $\mathcal{C}_{B}$.

1. Compute the discriminant $D(x)$ of $f(x, y)$ with respect to $y$ and determine its real roots within $B: x_{l} \leq \alpha_{1}<\ldots<\alpha_{s-1} \leq x_{r}$. Suppose $\alpha_{1} \neq x_{l}, \alpha_{s-1} \neq x_{r}$ and let $\alpha_{0}=x_{l}, \alpha_{s}=x_{r}$

2. For every $\alpha_{i}, 0 \leq i \leq s$, compute within $B$ the real roots of $f\left(\alpha_{i}, y\right), \beta_{i, 0}<\ldots<\beta_{i, t_{i}}$.

3. At each point $P_{i, j}=\left(\alpha_{i}, \beta_{i, j}\right)$, count the numbers of branches of $\mathcal{C}_{B}$ to the right and to the left.

4. For each $0 \leq i<s$, the number of branches to the right of points $P_{i, j}$ must be the same as the number of branches to the left of points $P_{i+1, j}$. Connect the points $P_{i, j}$ to the other endpoint of the segment with edges, obeying the branch counts and get the graph $\mathcal{G}_{T}$.

5. Label the generated curve segments from left to right $(i)$, top to bottom $(j)$ as

$$
\mathcal{S}_{T}=\left\{S_{i, j}, 1 \leq i \leq s, 1 \leq j \leq s_{i}\right\}
$$

where $s_{i}$ denotes the number of curve segments of $\mathcal{C}_{B}$ with $x$ in the interval $\left(\alpha_{i-1}, \alpha_{i}\right)$.

Let $V=\left\{\left(\alpha_{i}, \beta_{i, j}\right), 0 \leq i \leq s, 0 \leq j \leq t_{i}\right\}$ and decompose it into $V=V_{V} \cup V_{S} \cup V_{O}$, where $V_{V}$ is the set of vertical points, $V_{S}$ is the set of singular points, and $V_{O}$ is the other simple points.

Consider the curve defined by the following equation

$$
\mathcal{C}_{0}: f_{0}(x, y)=2 x^{4}-3 x^{2} y+y^{2}-2 y^{3}+y^{4}=0 .
$$

The figure and the corresponding topology graph of $\mathcal{C}_{0}$ is shown in Figure 1.
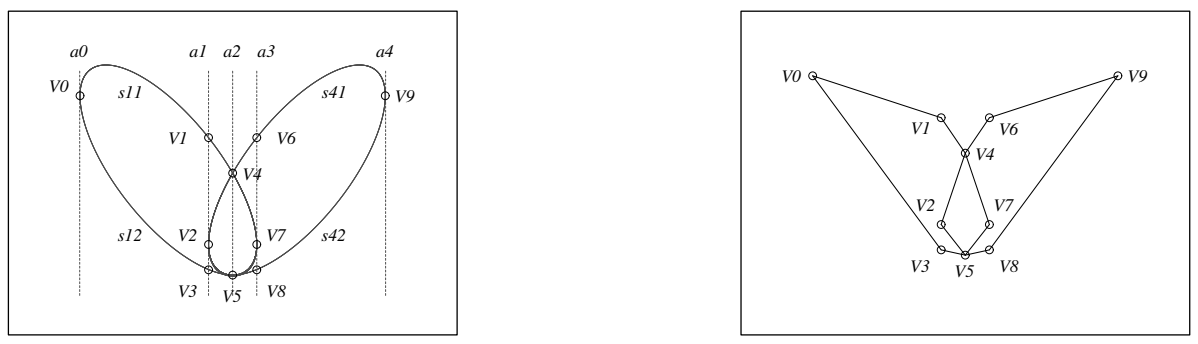

Fig. 1. A curve and its topology graph

With the topology $\mathcal{S}_{T}$ for $\mathcal{C}_{B}$, we can do the following basic operations for curve segments.

Algorithm 2.3 The inputs are a segment $S_{i_{0}, j_{0}}=S\left[P_{0}, P_{2}\right]$ in $\mathcal{S}_{T}$ for $\mathcal{C}_{B}$ with $P_{i}=\left(x_{i}, y_{i}\right)$ and an $\bar{x} \in\left(x_{0}, x_{2}\right)$. The output is a point $\bar{P}=(\bar{x}, \bar{y})$ on $S$.

1. Let $g(y)=f(\bar{x}, y)$ and find all the solutions $y_{1}>\ldots>y_{s}$ of $g(y)=0$ within $B$. Note that $g(y)=0$ has no repeated roots. 
2. Since $S_{i_{0}, j_{0}}$ is the $j_{0}$-th segment of $\mathcal{C}_{B}$ in the interval $\left(x_{0}, x_{2}\right)$ from top to bottom, $\bar{P}=\left(\bar{x}, y_{j_{0}}\right)$ should be on $S_{i_{0}, j_{0}}$.

Algorithm 2.4 The inputs are a point $\bar{P}=(\bar{x}, \bar{y})$ on $\mathcal{C}_{B}$ and the segment set $\mathcal{S}_{T}$ of $\mathcal{C}_{B}$. The output is a pair of footnotes $\left(i_{0}, j_{0}\right)$ such that $\bar{P}$ is on segment $S_{i_{0}, j_{0}} \in \mathcal{S}_{T}$.

1. Select a unique $\alpha_{i_{0}}$ such that $\alpha_{i_{0}-1} \leq \bar{x}<\alpha_{i_{0}}$. If there exists only one segment $S_{i_{0}, j_{0}}$ in the interval $\left(\alpha_{i_{0}-1}, \alpha_{i_{0}}\right)$, output $\left(i_{0}, j_{0}\right)$.

2. If each segment $S_{i_{0}, j}$ in the interval $\left(\alpha_{i_{0}-1}, \alpha_{i_{0}}\right)$ is triangle convex and there exists just one segment $S_{i_{0}, j_{0}}$ with $\bar{P}$ contained in its control triangle, output $\left(i_{0}, j_{0}\right)$.

3. Let $g(y)=f(\bar{x}, y)$ and isolate all the real roots of $g(y)=0$ within $B: y_{1}>\ldots>y_{r}$. Let $y_{j_{0}}$ be the one nearest to $\bar{y}$. Then output $\left(i_{0}, j_{0}\right)$.

\subsection{Flex computation and generation of triangle convex segments}

The cusps and flexes on rational curves are computed in [15]. A method to compute the real inflection points of cubic plane algebraic curves is given in [5]. There seems no work on computing the flexes of general implicit algebraic curves. Since this is not the central topics of this paper, we compute the flexes of $\mathcal{C}_{B}$ directly from its definition by solving the equation system $f(x, y)=0$ and $H(f)=0$ with well known methods based on resultant computation. Let $V_{F}$ be the set of the flexes in $\mathcal{C}_{B}$.

Algorithm 2.5 (Division at Flex) The inputs are $\mathcal{S}_{T}$ and $V_{F}$. The output is a set of triangle convex segments $\mathcal{S}_{F}=\left\{S_{i, j, k}, 1 \leq i \leq s, 1 \leq j \leq s_{i}, 1 \leq k \leq s_{i j}\right\}$ and its corresponding graph $\mathcal{G}_{F}$.

1. For each $S_{i, j} \in \mathcal{S}_{T}$, find all the points in $V_{F} \cap S_{i, j}$ with Algorithm 2.4. List these points from left to right according to the $x$ coordinate: $P_{i, j, k}, 1 \leq k \leq s_{i j}-1$.

2. Divide the segment $S_{i, j}$ at the points $P_{i, j, k}, 1 \leq k \leq s_{i j}-1$, ending with the curve segments $S_{i, j, k}, 1 \leq k \leq s_{i j}$.

3. If there is no flex on $S_{i, j}$, let $S_{i, j, 1}=S_{i, j}$ and $s_{i j}=1$.

4. Let $\mathcal{S}_{F}=\left\{S_{i, j, k}, 1 \leq i \leq s, 1 \leq j \leq s_{i}, 1 \leq k \leq s_{i j}\right\}$ and $\mathcal{G}_{F}=\mathcal{U}\left(\mathcal{S}_{F}\right)$. It is clear that $\mathcal{S}_{F}$ takes $V=V_{V} \cup V_{S} \cup V_{O} \cup V_{F}$ as the endpoints of its segments.

Theorem 2.6 Each segment $\mathcal{S}\left[P_{0}, P_{2}\right]$ in $\mathcal{S}_{F}$ is triangle convex.

Proof. Let $k(P)$ be the slope of the tangent line of $\mathcal{S}$ at point $P$ on $\mathcal{S}$. Since there is no cusp points, flexes and vertical points on $\mathcal{S}, k(P)$ must be monotonic and continuous. It could happen that $k\left(P_{0}\right)$ and $k\left(P_{2}\right)$ are equal to $\pm \infty$. Without loss of generality, suppose $k\left(P_{0}\right)>k\left(P_{2}\right)$. Then, there exist four possible cases as shown in Figure 2. In the first three cases, the right tangent line at $P_{0}$ and the left tangent line at $P_{2}$ have an intersection point, say $P_{1}$. Since the slope $k(P)$ is decreasing starting from $P_{0}$, the segment is below the line $P_{0} P_{1}$. Similarly, the segment is also below the line $P_{1} P_{2}$. To show that the segment is above line $P_{0} P_{2}$, let $p^{\prime}$ be a point on $\mathcal{S}$ between $P_{0}$ and $P_{2}$ such that $k\left(P^{\prime}\right)=0$. If such a $P^{\prime}$ 
does not exist, let $P^{\prime}=P_{2}$. Then from $P_{0}\left(P_{2}\right)$ to $P^{\prime}$ the distance from $\mathcal{S}$ to line $P_{0} P_{2}$ is increasing. This fact implies that the segment is above line $P_{0} P_{2}$. Therefore, the segment is inside triangle $P_{0} P_{1} P_{2}$. Since there exists no flexes and no cusp points, the convexity of the curve segment does not change and hence the segment forms a convex region with line segment $P_{0} P_{2}$. The fourth case can be proved similarly.
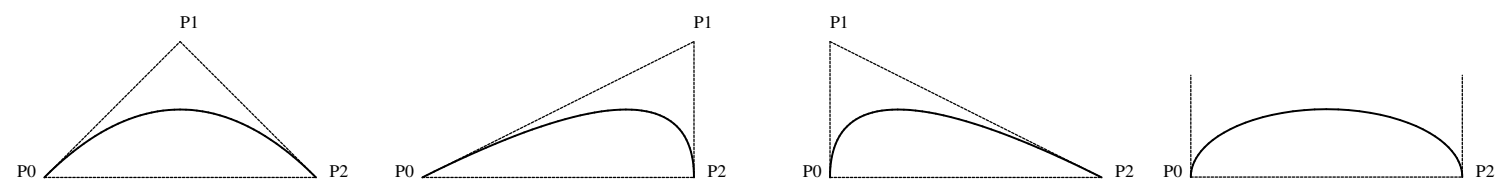

Fig. 2. Triangle convex segments

The $C_{0}$ in Figure 1 does not have flexes. Then its topology graph need not to be modified. The curve in Figure 14 has a flex point $v_{3}$.

\subsection{Tangent direction computation}

The tangent direction of a segment at a simple point can be easily obtained from its definition. We give a method to compute the tangent directions at a singular point.

Let $K$ be an algebraic closed field, and $\mathcal{C}$ a curve defined by $f(x, y)=0$ over $K$. Suppose that all derivatives of $f(x, y)$, up to and including $(r-1)$-th, vanish at $P_{0}$ but that at least one $r$-th derivative does not vanish. The tangent directions $(\lambda, \mu)$ to $\mathcal{C}$ at $P_{0}$, correspond to the roots of

$$
g(\lambda, \mu)=f_{x^{r}} \lambda^{r}+\left(\begin{array}{l}
r \\
1
\end{array}\right) f_{x^{r-1} y} \lambda^{r-1} \mu+\ldots+\left(\begin{array}{l}
r \\
r
\end{array}\right) f_{y^{r}} \mu^{r}=0
$$

where all the partial derivatives are evaluated at $P_{0}$. But for a real algebraic curve defined by $f(x, y) \in \mathcal{R}[x, y]$, there does not exist such a one-one correspondence between the real roots of the equation $g(\lambda, \mu)=0$ and the tangent directions of the components containing $P_{0}$. For example, let the plane curve $\mathcal{C}$ defined by

$$
f(x, y)=y^{3}-2 y^{2} x+15 y x^{4}-x^{5} .
$$

The real roots of $g(\lambda, \mu)$ at $P_{0}=(0,0)$ are $(1,0)$ and $(1,2)$. However, the curve has no real component with the tangent direction $(1,0)$ at $P_{0}$ (Figure 3 ). In fact, the set of the tangent directions $(\lambda, \mu)$ of all real components containing $P_{0}$ is just a subset of the real roots set of $g(\lambda, \mu)=0$.

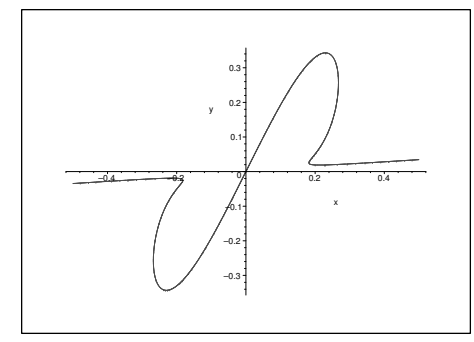

Fig. 3. Unexpected tangent direction 
Algorithm 2.7 The input is the segment set $\mathcal{S}_{F}$. The output is a set of segments $\mathcal{S}_{\vec{F}}$ with the tangent directions at the endpoints of each segment and its corresponding graph $\mathcal{G}_{\vec{F}}$.

1. Let $V=V_{V} \cup V_{S} \cup V_{O} \cup V_{F}$ be the endpoints of the segments in $\mathcal{S}_{F}$.

2. For every point $P_{0}=\left(x_{0}, y_{0}\right)$ in $V_{S}$ compute the left tangent directions for the segments $S_{j}, j=0, \ldots, r$ with $P_{0}$ as the left endpoint. We assume that $S_{0}, \ldots, S_{r}$ are from top to bottom.

- Find all the solutions $\left(\lambda_{i}, \mu_{i}\right), 0 \leq i \leq s$, of the homogeneous algebraic equation $g(\lambda, \mu)=0$ defined in (3). Let $k_{i}=\frac{\mu_{i}}{\lambda_{i}}$ with $k_{i}=+\infty$ when $\lambda_{i}=0$ and $\mu_{i}>0$ and $k_{i}=-\infty$ when $\lambda_{i}=0$ and $\mu_{i}<0$.

- Sort $k_{i}$ in a descending order and rename them as $k_{i}, 0 \leq i \leq s$.

- Select a $\bar{\delta}<\delta$ such that $I_{i} \cap I_{i+1}=\phi, 0 \leq i \leq s-1$, where $I_{i}=\left[k_{i}-\bar{\delta}, k_{i}+\bar{\delta}\right], 0 \leq$ $i \leq s$. If $k_{s}=+\infty$, set $\bar{k}_{s}=\max \left(0,2 k_{s-1}\right)$ and $I_{s}=\left[\bar{k}_{s},+\infty\right)$; if $k_{0}=-\infty$, set $\bar{k}_{s}=\min \left(0,2 k_{1}\right)$ and $I_{0}=\left(-\infty, \bar{k}_{0}\right]$.

- Set $\epsilon=\frac{x_{1}-x_{0}}{100}$, where $x_{1}$ is the minimal $x$ coordinates of the right endpoints of $S_{j}$.

- Find a point $\left(x_{0}+\epsilon, \overline{y_{j}}\right)$ on $S_{j}$ with Algorithm 2.3. Let $\bar{k}_{j}=\frac{\overline{y_{j}}-y_{0}}{\epsilon}$, which could be considered as an approximation to the slope of $S_{j}$. If there exists a $\bar{k}_{j}$ which is not in $\bigcup I_{i}$, set $\epsilon:=\epsilon / 10$ and repeat this step. This step will end because $\bar{k}_{j}$ is approaching to the slope of some segment at point $P_{0}$.

- Suppose that $\bar{k}_{j}$ is in $I_{n_{j}}$. Then the left tangent direction of $S_{j}$ is $\left(\lambda_{n_{j}}, \mu_{n_{j}}\right)$.

3. Compute the right tangent directions $T_{+}$in a similar way by taking $-\epsilon$ instead of $\epsilon$.

4. Compute the left tangent and right tangent directions at the points $V \backslash V_{S}$ according to the definition.

5. Add the tangent information to each segment in $\mathcal{S}_{F}$ to obtain $\mathcal{S}_{\vec{F}}$. Set $\mathcal{G}_{\vec{F}}=\mathcal{U}\left(\mathcal{S}_{\vec{F}}\right)$.

\subsection{Segment combination}

We will combine some segments under the condition that the triangle convexity of the segments will be kept.

Algorithm 2.8 The input is $\mathcal{S}_{\vec{F}}$. The output is a set of segments $\mathcal{S}_{\vec{C}}$ for $\mathcal{C}_{B}$ with less segments than that of $\mathcal{S}_{\vec{F}}$.

1. Let the endpoints of the segments in $\mathcal{S}_{\vec{F}}$ be $V\left(\mathcal{S}_{\vec{F}}\right)=V_{V} \bigcup V_{S} \cup V_{O} \cup V_{F}$

2. For all $P_{0} \in V_{O}$ and segments $S_{i_{0}, j_{0}, k_{0}}\left[P_{1}, P_{0}\right], S_{i_{1}, j_{1}, k_{1}}\left[P_{0}, P_{2}\right]$, combine them as one segment $S_{J}$ where $J=\left\{\left\{i_{0}, j_{0}, k_{0}\right\},\left\{i_{1}, j_{1}, k_{1}\right\}\right\}$. The information in $J$ is needed, e.g. in Algorithm 2.3.

3. Keeping those segments containing no points in $V_{O}$ unchanged, we obtain $\mathcal{S}_{\vec{C}}$. Let $\mathcal{G}_{\vec{C}}=\mathcal{U}\left(\mathcal{S}_{\vec{C}}\right)$. We have $V\left(\mathcal{G}_{\vec{C}}\right)=V_{V} \cup V_{S} \cup V_{F}$. 

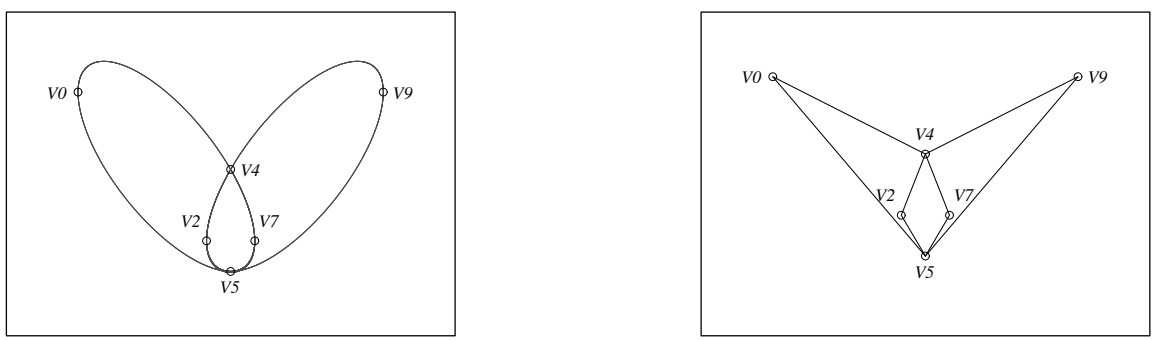

Fig. 4. Segment comb. and the graph-1

Since only two segments sharing a simple point are combined, the topology of the graph does not change. Figure 4 shows the combined segments and its corresponding graph of the curve in Figure 1.

The following algorithm tries to combine segments at singular points which are intersections of two curve branches enuring that the resulted approximate curves have the same topology with the original curve.

Algorithm 2.9 The input is the same with Algorithm 2.8. The output is a refined plane graph $\tilde{\mathcal{G}}_{\vec{C}}$ such that each segment in $\mathcal{S}_{\vec{C}}=\mathcal{U}^{-1}\left(\tilde{\mathcal{G}}_{\vec{C}}\right)$ is triangle convex.

1. Simplify $\mathcal{G}_{\vec{F}}$ to $\tilde{\mathcal{G}}_{\vec{C}}$ with Algorithm 2.8 .

2. Let $V_{f} \subset V_{S}$ be the set of singular points with degree (in the graph) less than or equal to four and not all the left or right tangent directions at the point are the same.

3. For a point $P_{S} \in V_{f}$ let $E_{S}$ be the set of edges in $\overline{\mathcal{G}}_{\vec{C}}$ with $P_{S}$ as an endpoint. If $E_{S}$ is empty, go to step 7 .

4. Find two segments $E_{i_{0}, j_{0}, k_{0}}\left[P_{\alpha}, T_{\alpha}, P_{S}, T_{S}\right]$ and $E_{i_{1}, j_{1}, k_{1}}\left[P_{S}, T_{S}, P_{\beta}, T_{\beta}\right]$ in $E_{S}$ such that they are at the same side of the line passing through $P_{S}$ and with direction $T_{S}$. In Figure 5 the left figure satisfies this condition while the right one does not.
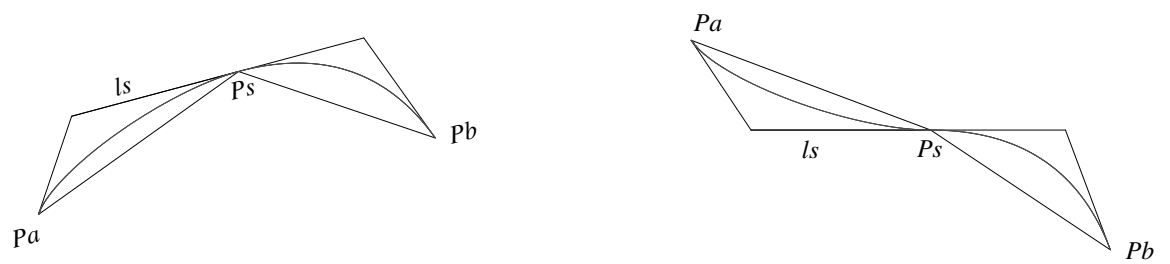

Fig. 5. Convexity maintenance

5. Combine the edges $E_{i_{0}, j_{0}, k_{0}}$ and $E_{i_{1}, j_{1}, k_{1}}$ into a new edge and refine $\overline{\mathcal{G}}_{\vec{C}}$ as step 2 in Algorithm 2.8.

6. Let $E_{S}=E_{S} \backslash\left\{E_{i_{0}, j_{0}, k_{0}}, E_{i_{1}, j_{1}, k_{1}}\right\}$ and go to step 4 until no such segments exist.

7. Let $V_{f}=V_{f} \backslash\left\{P_{S}\right\}$ go to step 3 until $V_{f}$ is empty. 
8. Set $\bar{S}_{\vec{C}}=\mathcal{U}^{-1}\left(\overline{\mathcal{G}}_{\vec{C}}\right)$.

Figure 6 shows the combined segments and its corresponding graph of the curve in Figure 1 with Algorithm 2.9.
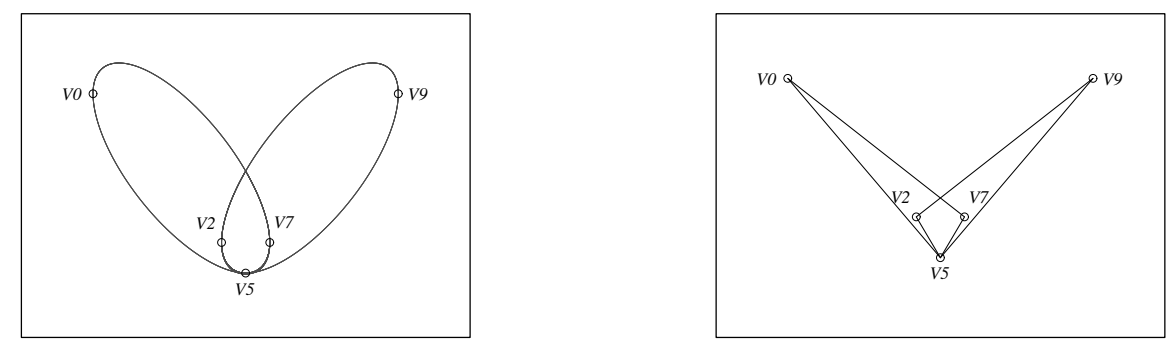

Fig. 6. Segments comb. and the graph-2

\section{Segment approximation}

An algorithm will be shown to approximate a triangle convex segment in this section. It mainly consists of two parts: the shoulder point computation and the segment approximation.

\subsection{Quadratic rational Bézier curve}

Any quadratic segment can be expressed by a rational quadratic Bézier curve with the following form [7, 14],

$$
P(\omega, t)=\frac{P_{0} \phi_{0}(t)+\omega P_{1} \phi_{1}(t)+P_{2} \phi_{2}(t)}{\phi_{0}(t)+\omega \phi_{1}(t)+\phi_{2}(t)} ; 0 \leq t \leq 1,
$$

where $\omega \in \mathcal{R}, P_{i} \in \mathcal{R}^{2}$, and $\phi_{i}, 0 \leq i \leq 2$, are the quadratic Bernstein basis [7, 14]. The quadratic Bézier curve (4) has the following properties $[7,14]$.

(P1) Convex Hull: The segment $P(\omega, t)$, lies in its control triangle $P_{0} P_{1} P_{2}$ for $\omega>0$, and is triangle convex.

(P2) Endpoints Interpolation: $P(t)$ passes through the endpoints $P_{0}, P_{2}$ with the corresponding tangent directions $P^{\prime}(0)$ and $P^{\prime}(1)$ parallel to $P_{0} P_{1}$ and $P_{1} P_{2}$.

(P3) Control Point at Infinity: If the tangent lines at the endpoints are parallel, it can be written as below

$$
P(\omega, t)=\frac{P_{0} \phi_{0}(t)+\omega T \phi_{1}(t)+P_{2} \phi_{2}(t)}{\phi_{0}(t)+\phi_{2}(t)} ; \quad 0 \leq t \leq 1,
$$

where $T$ is the tangent vector at either of the endpoints.

(P4) Shoulder Point: The point $S_{P}=P\left(\frac{1}{2}\right)$ is called the shoulder point of $P(t)$, which can be computed from

$$
S_{P}=\frac{1}{2}\left(Q_{0}+Q_{2}\right)
$$

where $Q_{0}=\frac{P_{0}+\omega P_{1}}{1+\omega}, Q_{2}=\frac{\omega P_{1}+P_{2}}{1+\omega}$, or $Q_{0}=P_{0}+\omega T, Q_{2}=\omega T+P_{2}$, when (5) is used. $S_{P}$ is the unique point in the curve $P(t), 0 \leq t \leq 1$, that has the maximum distance to $P_{0} P_{2}$.

Let $S\left[P_{0}, T_{0}, P_{2}, T_{2}\right]$ be a triangle convex curve segment, and $P_{1}$ the intersection point of the tangent lines at $P_{0}$ and $P_{2}$ if it exists. The curve family $P(\omega, t)$ with $\omega>0$ in (4) 


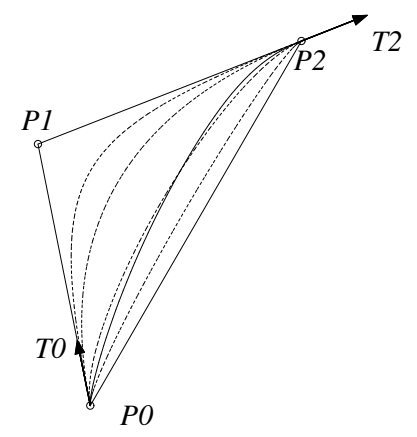

Fig. 7. Approximation curve family

interpolates points $P_{0}, P_{2}$ and with tangent directions $T_{0}, T_{2}$ at $P_{0}, P_{2}$, and thus provides a $G^{1}$ approximation to $S$. Suppose that the solid curve in Figure 7 is the curve segment $S$ and the dotted curves are the quadratic curve family $P(\omega, t)$. In the next section, we give a method called shoulder point approximation to select a proper value for $\omega$ so that it has an optimal approximation to the segment.

\subsection{Shoulder point computation}

From the definition of the shoulder point, we can see that the gradient $\left(f_{x}, f_{y}\right)$ of $\mathcal{C}$ at the shoulder point $S_{P}$ of $S\left[P_{0}, P_{2}\right]$ is perpendicular to $\left(k_{0}, k_{1}\right)=P_{2}-P_{0}$. The following equations system is therefor to be solved to get $S_{P}$

$$
F(x, y):\left\{\begin{array}{l}
f(x, y)=0, \\
h(x, y)=k_{0} f_{x}+k_{1} f_{y}=0 .
\end{array}\right.
$$

Since the shoulder point is needed in each process of segment approximation, it should be computed in a fast way. The following algorithm provides a fast method to compute the shoulder point.

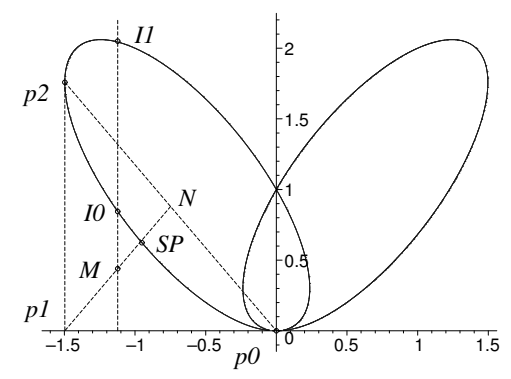

Fig. 8. Find a shoulder point

Algorithm 3.1 The input is a triangle convex segment $S\left[P_{0}, T_{0}, P_{2}, T_{2}\right]$. The output is the shoulder point $S_{P}$ of $S$ if it is found.

1. Select an initial point $I_{0}$. Let $N=\frac{P_{0}+P_{2}}{2}$. If $T_{0}$ is not parallel to $T_{2}$, let $M=\frac{N+P_{1}}{2}=$ $\frac{P_{0}+2 P_{1}+P_{2}}{4}$; otherwise let $M=N$. Let $M=\left(M_{x}, M_{y}\right)$. Find a point $I_{0}=\left(M_{x}, I_{y}\right)$ on $S$ with Algorithm 2.3. 


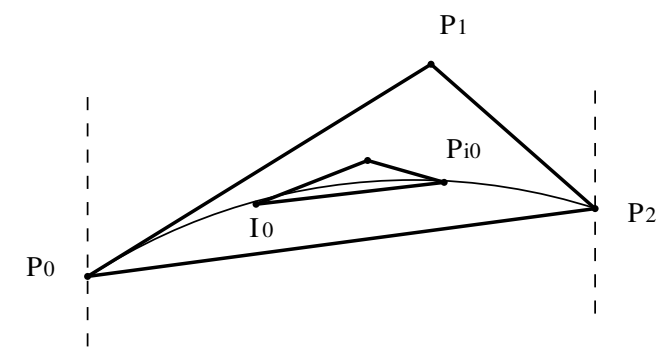

Fig. 9. Find a new initial point

2. Find the shoulder point with the Newton-Ralphson method. Starting at $p_{0}=I_{0}$, repeat the following process until $\left\|\Delta p_{k}\right\|<\delta$ is satisfied.

(a) Let $J(x, y)$ be the Jacobian matrix of $F(x, y)$.

(b) Solve the system of linear equations $J\left(p_{k}\right) \Delta p_{k}=-F\left(p_{k}\right)$.

(c) Let $p_{k+1}:=p_{k}+\Delta p_{k}$. If $p_{k+1}$ is between the two tangent lines at the endpoints of $S$, goto the preceding step. Otherwise, the algorithm fails.

3. If $p_{k+1}$ is got, output $S_{P}=p_{k+1}$. Otherwise, the algorithm fails.

In Figure $8, I_{0}$ is the initial position and $S_{P}$ is the shoulder point computed with the NewtonRalphson method.

The above algorithm can be used to compute the shoulder point in most cases. If it fails, the following algorithm tries to refine the initial value of the Newton-Ralphson method until a shoulder point is obtained.

Algorithm 3.2 The input and output are the same with that of Algorithm 3.1 and suppose $P_{i}=\left(x_{i}, y_{i}\right), i=0,2$.

1. Use Algorithm 3.1 to find a shoulder point. If it fails, goto the next step.

2. Let $I_{0}=\left(I_{x}, I_{y}\right)$ be the initial point used in the preceding step, and $l(x, y)=0$ the line pass through $I_{0}$ and parallel to $P_{0} P_{2}$ with the form $y=I_{y}+\frac{y_{2}-y_{0}}{x_{2}-x_{0}}\left(x-I_{x}\right)$. Substitute $y$ into $f(x, y)=0$ and we get a univariate equation $g(x)=0$.

3. Find all the solutions $\bar{x}_{0}<\ldots<\bar{x}_{m}$ of $g(x)=0$ in the interval $\left(x_{0}, x_{2}\right)$. Let $\bar{P}_{i}=$ $\left(\bar{x}_{i}, I_{y}+\frac{y_{2}-y_{0}}{x_{2}-x_{0}}\left(\bar{x}_{i}-I_{x}\right)\right), 0 \leq i \leq m$.

4. Select a unique point $\bar{P}_{i_{0}}$ with Algorithm 2.4 such that it is on $S$ (Figure 9). Set $P_{0}:=I_{0}, P_{2}:=\bar{P}_{i_{0}}$ and go to step 1 .

The geometric meaning of the algorithm is shown in Figure 9. Since the initial positions converge to the shoulder point, the process will end successfully. 


\subsection{Segment approximation}

Let $P=(x(t), y(t)), t \in[0,1]$ be a parametric curve and $S\left[P_{0}, P_{2}\right]$ a curve segment for $f(x, y)=0$. The distance from $P=(x(t), y(t))$ to a curve $f(x, y)=0$ can be approximated by the following approximation error function $[6,16]$,

$$
e(t)=\frac{f(x(t), y(t))}{\left[\left(f_{x}(x(t), y(t))^{2}+f_{y}(x(t), y(t))^{2}\right)^{2}\right]^{\frac{1}{2}}} .
$$

The approximation error can then be set as the following optimization problem,

$$
e=\max _{0 \leq t \leq 1}(e(t))
$$

In practice, we sample $t$ as $t_{i}=\frac{i}{n}, i=0, \cdots, n$, for a proper value of $n$, say $n=20$, and set the approximation error as $\max \left(\left|d\left(t_{i}\right)\right|\right)$.

Let $S\left[P_{0}, T_{0}, P_{2}, T_{2}\right]$ be a triangle convex segment, and $\delta$ a small positive number. By a $\delta$-approximation for $S$, we mean a sequence of points $\bar{P}_{i}=\left(\bar{x}_{i}, \bar{y}_{i}\right), 0 \leq i \leq m$, satisfying $\bar{P}_{0}=P_{0}, \bar{P}_{m}=P_{2}, \bar{x}_{0}<\bar{x}_{1}<\ldots<\bar{x}_{m}$ and rational quadratic Bézier curve segments $P_{i}(t), 1 \leq i \leq m$, such that

- $P_{i}(t), i=1, \cdots, m$ pass through $\bar{P}_{i-1}, \bar{P}_{i}$ and have the same tangent directions with $S$ at these points; and

- the approximation error between $S$ and $P_{i}(t)$ is less than $\delta$ with $t$ varying in the interval $(0,1)$.

Algorithm 3.3 (Segment approximation) The inputs are a triangle convex curve segment $S\left[P_{0}, T_{0}, P_{2}, T_{2}\right]$ and a small positive number $\delta$. The output is a $\delta$-approximation to $S$.

1. Let $P_{i}=\left(x_{i}, y_{i}\right), i=0,1,2$ and $T_{0}=\left(t_{x}, t_{y}\right)$.

2. According to the interpolating requirements at the endpoints of $P(\omega, t)$, set $P(\omega, t)$ as (4), or (5) if $T_{0}$ and $T_{2}$ are parallel.

3. Find the shoulder point $S_{P}=\left(P_{x}, P_{y}\right)$ on $S$ with Algorithm 3.2.

4. Let the shoulder point of $P(\omega, t)$ be $S(\omega)$, as expressed in equation (6). A specific value $\omega_{0}$ will be determined such that $S\left(\omega_{0}\right)$ has a minimum distance to the shoulder point $S_{P}$.

If $T_{0}$ is not parallel to $T_{2}$, we have

$$
\begin{aligned}
S(\omega) & =\left(S_{x}, S_{y}\right)=\frac{P_{0}+2 \omega P_{1}+P_{2}}{2(1+\omega)} \\
& =\left(\frac{x_{0}+2 \omega x_{1}+x_{2}}{2(1+\omega)}, \frac{y_{0}+2 \omega y_{1}+y_{2}}{2(1+\omega)}\right) .
\end{aligned}
$$

Solving the equation, $\frac{\partial d^{2}(P, S(\omega))}{\partial \omega}=0$, where $d^{2}\left(S_{P}, S(\omega)\right)=\left(P_{x}-S_{x}\right)^{2}+\left(P_{y}-S_{y}\right)^{2}$, we get

$$
\omega_{0}=\frac{1}{2} \cdot \frac{\left(x_{0}+x_{2}-2 P_{x}\right)+\alpha\left(y_{0}+y_{2}-2 P_{y}\right)}{\left(P_{x}-x_{1}\right)+\alpha\left(P_{y}-y_{1}\right)}
$$


where $\alpha=\frac{y_{0}+y_{2}-2 y_{1}}{x_{0}+x_{2}-2 x_{1}}$. If $T_{0}$ is parallel to $T_{2}$, we get in a similar way

$$
\omega_{0}=\frac{\left(2 S_{x}-x_{0}-x_{2}\right)+\left(2 S_{y}-y_{0}-y_{2}\right)}{2\left(t_{x}^{2}+t_{y}^{2}\right)} .
$$

5. Compute the approximate error $e$ with equation (9).

6. If $e<\delta$, end this procedure. Otherwise, divide the segment into two parts at the point $S_{P}$ and repeat the approximation method for them until the approximation error is less than $\delta$.

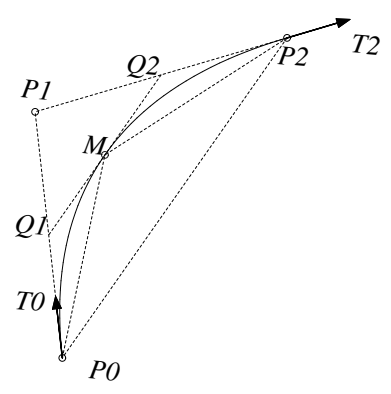

Fig. 10. Error control

The algorithm is ensured to be terminable for any small positive number $\delta$ from the theorem below.

Theorem 3.4 With Algorithm 3.3, the approximation error is convergent to zero.

Proof. See also Figure 10. Suppose that we get the approximation curve $P(t)$ for a curve segment $S$ after one step of approximation. We have that $P(t)$ and $S$ are contained in the same triangles $P_{0} P_{1} P_{2}$. After one step of recursive segmentation, the curves are contained in triangle $P_{0} Q_{1} M$ and $P_{2} Q_{2} M$ respectively. The area between the two curves is restricted by the area of the control triangles. The distance error of the two curves must be less than the height of the triangle. After a sufficient number of segmentation, the error will become as small as possible.

Figure 11 shows an approximation spline for the curve $\mathcal{C}_{1}$ defined as follows:

$$
\mathcal{C}_{1}:\left(x^{2}+y^{2}\right)^{3}-4 x^{2} y^{2}=0 .
$$

The approximation process is as follows. $\mathcal{C}_{1}$ is first approximated with a segment $S_{0}(t)$, with the approximation error function $e_{0}$ (expressed as (9)) shown in Figure 12. $\mathcal{C}_{1}$ is then divided at its shoulder point $V_{1}$ and the two segments are approximated by $\mathcal{C}_{00}$ and $\mathcal{C}_{01}$ with approximation error functions $e_{00}$ and $e_{01}$. Due to the symmetry of $\mathcal{C}_{0}$, we only show $e_{00}$ in Figure 12. In a similar way, $e_{000}, e_{001}, e_{0010}, e_{0011}$ are obtained and shown in Figure 12.

Figure 13 shows the resulted approximations and error functions for the curve $C_{0}$ defined in $(2)$. 


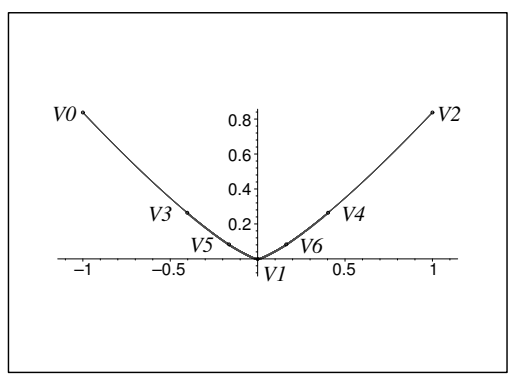

Fig. 11. Appr. segments of $\mathcal{C}_{1}$
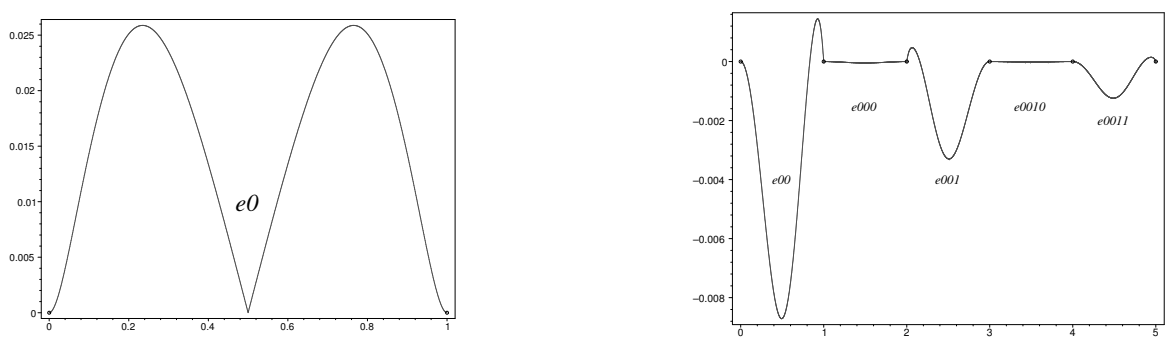

Fig. 12. Approximation error of $C_{1}$

\section{Curve tracing}

The following concepts from graph theory will be used [4]. A trail is a sequence of distinct edges $e_{1}, e_{2}, \ldots, e_{k}$ such that $e_{i}$ and $e_{i+1}$ share a common vertex. A trail that traverses every edge of $\mathcal{G}$ is called an Euler trail of $\mathcal{G}$.

Theorem 4.1 [4] In any graph, the number of vertices of odd degree is even.

Theorem 4.2 [4] A connected graph has an Euler trail if and only if it has at most two vertices of odd degree.

To guarantee the $G^{1}$ continuity, two segments $S\left[P_{1}, T_{1}, P_{2}, T_{2}\right]$ and $S\left[P_{3}, T_{3}, P_{4}, T_{4}\right]$ can be connected in a trail only if $P_{2}=P_{3}$ and $T_{2}=\lambda T_{3}$ for a non-zero number $\lambda$. A trail in the topology graph of a curve is called a branch if the corresponding segments of every neighboring edges in the trail satisfy the above property.

By private communication with Guangxing Zeng, we know that ${ }^{2)}$

Lemma 4.3 Every point on a real plane algebraic curve has an even number of segments originating from it.

As a consequence of this result, the vertices in the graph $\mathcal{G}_{\vec{C}}$ with odd degrees must be the intersection of the curve with the boundaries of $B$, which are called boundary points. Thus, there are two kinds of trails for $\mathcal{G}_{\vec{C}}$ : closed Euler trails for a closed component of $\mathcal{C}_{B}$ and trails from a boundary point to another boundary point.

\footnotetext{
${ }^{2)}$ This result makes our algorithm clearer, but is not necessary for our algorithm.
} 

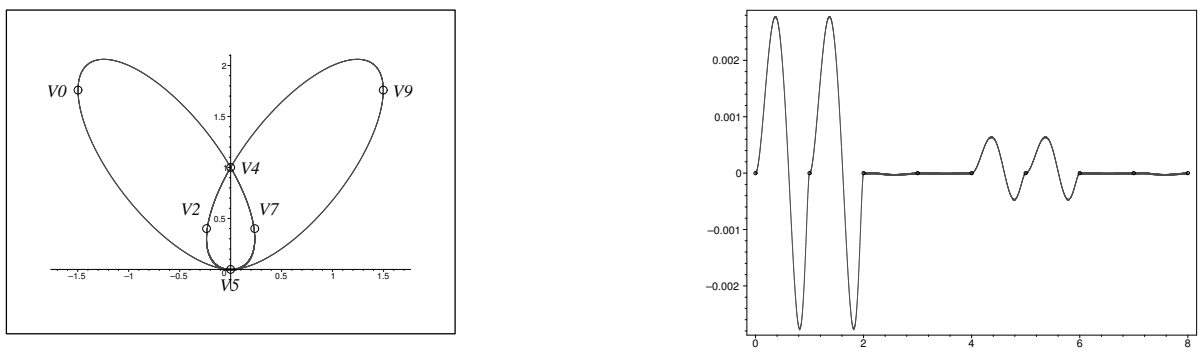

Fig. 13. Approximation of $\mathcal{C}_{0}$

Algorithm 4.4 The input is a graph $\mathcal{G}_{\vec{C}}$ for a plane curve $\mathcal{C}_{B}$. The outputs are edge-disjoint branches $B_{i}, 1 \leq i \leq r$ in $\mathcal{G}_{\vec{C}}$ such that $\bigcup_{i=1}^{r} B_{i}=E\left(\mathcal{G}_{\vec{C}}\right)$.

1. Decompose $\mathcal{G}_{\vec{C}}$ into closed subgraphs $\overline{\mathcal{G}}_{i}, 1 \leq i \leq v$ and $\tilde{\mathcal{G}}_{i}, 1 \leq i \leq u$ such that there exists at least one vertex of odd degree in $\overline{\mathcal{G}}_{i}$.

2. For $1 \leq i \leq u$, try to generate a closed branch for $\overline{\mathcal{G}}_{i}$ as follows. If $\overline{\mathcal{G}}_{i} \cap V_{S}$ is not empty, select a point in it as $v_{0}$. Otherwise, select an arbitrary vertex in $\overline{\mathcal{G}}_{i}$ as $v_{0}$. Starting from $v_{0}$, use a breadth-first way to search all the vertices of $\overline{\mathcal{G}}_{i}$ to generate a branch $B$ with maximal length. Remove the edges in $B$ from $\overline{\mathcal{G}}_{i}$ to obtain a new graph $G^{\prime}$. if $G^{\prime}$ is empty, a closed branch is generated. Otherwise repeat step 1 for $G^{\prime}$.

3. Let $v_{0}$ be a boundary vertex in $\tilde{\mathcal{G}}_{i}$ with odd degree. Starting from $v_{0}$, generate a branch similar to step 2. Repeat this step until no boundary vertices exist.

4. Now there exist no vertices with odd degree in $\tilde{\mathcal{G}}_{i}$. If $\tilde{\mathcal{G}}_{i}$ is not empty, repeat step 1 for $\tilde{\mathcal{G}}_{i}$.

The tracing orders for the graphs in Figures 4 and 6 are $v_{5} v_{9} v_{4} v_{2} v_{5} v_{7} v_{4} v_{0} v_{5}$ and $v_{5} v_{9} v_{2} v_{5} v_{7} v_{0} v_{5}$ respectively.

\section{Main algorithm and experimental results}

Algorithm 5.1 The inputs are a plane curve $\mathcal{C}: f(x, y)=0$ with $f(x, y) \in Z[x, y]$ an irreducible polynomial of degree larger than two, a bounding box $B$, an error bound $\delta>0$. The outputs are several rational quadratic B-spline curves $B_{i}(t), 1 \leq i \leq r$, such that they give a $C^{1}$ approximation to $\mathcal{C}$ within the bounding box $B$ with the approximation error less than $\delta$.

1. Topology Determination. Determine the topology of $\mathcal{C}_{B}$ with Algorithm 2.2. Let the resulted segments be $\mathcal{S}_{T}$ and let $\mathcal{G}_{T}=\mathcal{U}\left(\mathcal{S}_{T}\right)$.

2. Flex Computation. Compute the set $V_{F}$ of the flexes of $\mathcal{C}_{B}$ as shown in section 2.3. Divide those segments in $\mathcal{S}_{T}$ containing flexes with Algorithm 2.5 to obtain a new set of segments $\mathcal{S}_{F}$ and let $\mathcal{G}_{F}=\mathcal{U}\left(\mathcal{S}_{F}\right)$. 

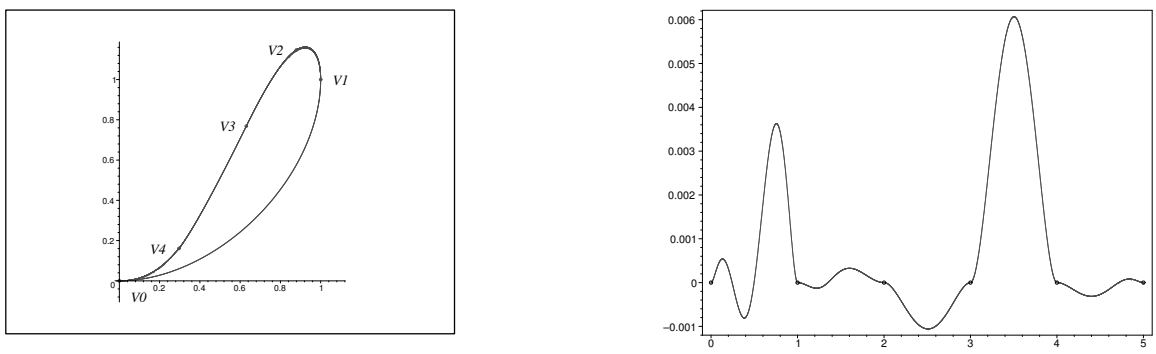

Fig. 14. Approximation of $\mathcal{C}_{2}$
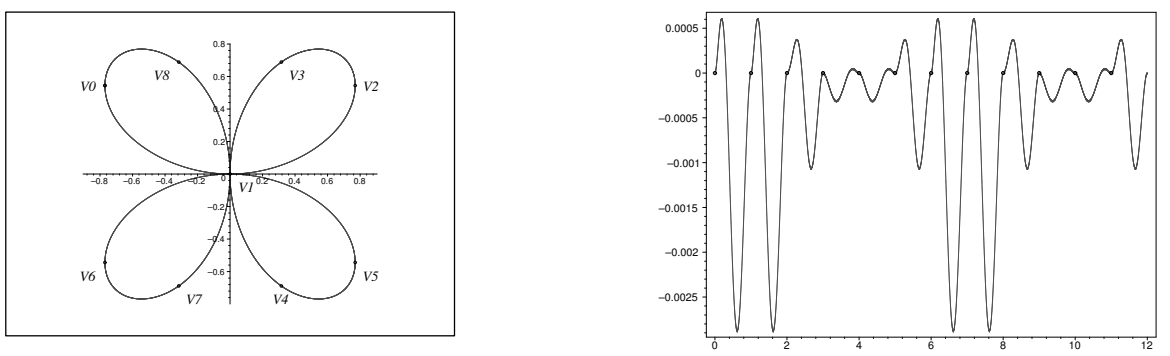

Fig. 15. Approximation of $\mathcal{C}_{3}$

3. Tangent Computation. Compute the tangent directions with Algorithm 2.7 to obtain $\mathcal{G}_{\vec{F}}$ and $\mathcal{S}_{\vec{F}}$.

4. Segment Combination. Combine some edges in the graph $\mathcal{G}_{\vec{F}}$ with Algorithm 2.9 to obtain a new graph $\mathcal{G}_{\vec{C}}$ and $\mathcal{S}_{\vec{C}}$.

5. Segment Approximation. Find a $\delta$-approximation rational quadratic Bézier curve for each segment in $\mathcal{S}_{\vec{C}}$ with Algorithm 3.3.

6. Curve Tracing. Find $r$ edge-disjoint branches $B_{i}, 1 \leq i \leq r$, with Algorithm 4.4. Let $E_{i}=\mathcal{U}^{-1}\left(B_{i}\right)$ be the corresponding curve branches in $\mathcal{C}_{B}$.

7. B-spline Conversion. These rational quadratic Bézier curves for the segments in $E_{i}$ are converted into a B-spline curve $C_{i}$ with a proper knot selection using the method in [3]. $C_{i}$ provides a $C^{1}$ approximation to branch $E_{i}$.

The method reported is implemented in Maple. The benchmark curves $\mathcal{C}_{0}, \mathcal{C}_{1}, \mathcal{C}_{2}, \mathcal{C}_{3}$ are from [21]. Curves $\mathcal{C}_{4}$ and $\mathcal{C}_{5}$ are taken from [11].

$$
\begin{aligned}
\mathcal{C}_{2}: & x^{4}+x^{2} y^{2}-2 x^{2} y-x y^{2}+y^{2}=0, \\
\mathcal{C}_{3}: & \left(x^{2}+y^{2}\right)^{3}-4 x^{2} y^{2}=0, \\
\mathcal{C}_{4}: & y^{8}+y^{7}-(8+7 x) y^{6}-\left(7-21 x^{2}\right) y^{5}-\left(-20-35 x+35 x^{3}\right) y^{4}-\left(-14+70 x^{2}-35 x^{4}\right) y^{3}- \\
& \left(16+42 x-70 x^{3}+21 x^{5}\right) y^{2}-\left(7-42 x^{2}+35 x^{4}-7 x^{6}\right) y+7 x-14 x^{3}+7 x^{5}-x^{7}=0, \\
\mathcal{C}_{5}: & -3+12 y^{2}+2 y^{4}-12 y^{6}+y^{8}+12 x^{2}-28 y^{2} x^{2}+12 y^{4} x^{2}+4 y^{6} x^{2}-18 x^{4}+20 y^{2} x^{4}+2 y^{4} x^{4}+ \\
& 12 x^{6}-4 x^{6} y^{2}-3 x^{8}=0 .
\end{aligned}
$$

In the figures in this section, the left figures show the approximation results and the right figures show the approximation error functions. Specifically, the curve in the right figures defined in the interval $(i, i+1)$ corresponds to the $(i-1)$-th curve segment in the left figures. 
Tracing order for $\mathcal{C}_{2}: v_{0} v_{1} v_{2} v_{3} v_{4} v_{0}$.

Tracing order for $\mathcal{C}_{3}: v_{0} v_{1} v_{2} v_{3} v_{1} v_{4} v_{5} v_{1} v_{6} v_{0}$.

Tracing order for $\mathcal{C}_{4}: v_{0} v_{1} v_{2} v_{3} v_{4} v_{5} v_{6} v_{7} v_{8}$.

Tracing order for $\mathcal{C}_{5}: v_{0} v_{1} v_{2} v_{3} v_{4} v_{5} v_{6} v_{7} v_{8}$ and $v_{9} v_{10} v_{11} v_{12} v_{9}$. This curve consists of four branches and is symmetric with the $x$ axis. We only show the result for two of the branches.
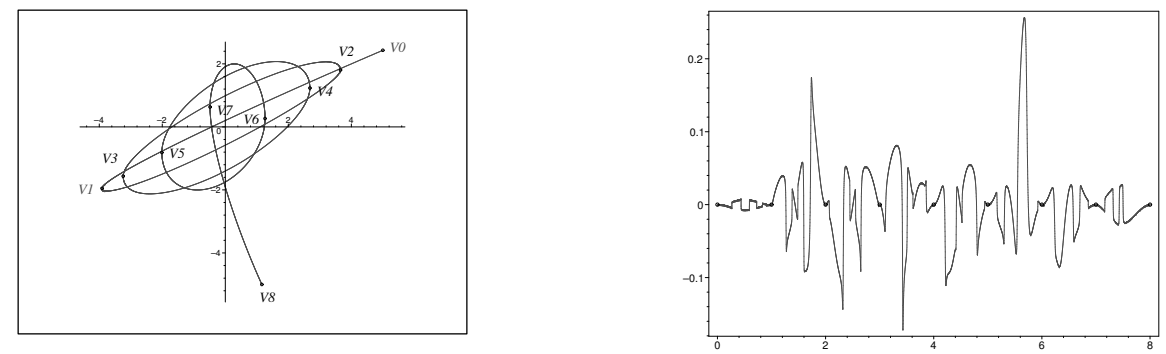

Fig. 16. Approximation of $\mathcal{C}_{4}$

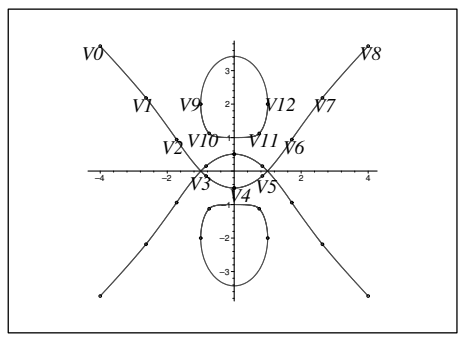

Fig. 17. Approximation of $\mathcal{C}_{5}$
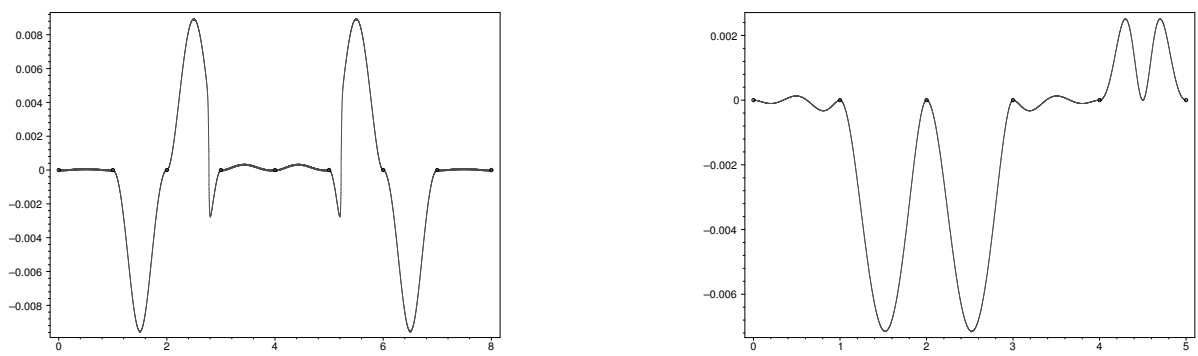

Fig. 18. Error function of $\mathcal{C}_{5}$

\section{Extension to spatial curves}

With the method we can also give a quadratic approximation to a spatial curve defined as the intersection of two surfaces in implicit form.

\subsection{Approximation to implicitly defined spatial curves}

Suppose that a spatial curve $S C:=\operatorname{Zero}(f, g)$, which is implicitly defined as the intersection of two surfaces $f(x, y, z)=0$ and $g(x, y, z)=0$. We first have the following theorem about $S C[8]$. 
Theorem 6.1 [8] We may find an inverse linear transformation $(x, y, z)=A\left(x^{\prime}, y^{\prime}, z^{\prime}\right)$ such that the new space curve $\mathcal{C}^{\prime}$ defined by $\bar{f}\left(x^{\prime}, y^{\prime}, z^{\prime}\right)=0$ and $\bar{g}\left(x^{\prime}, y^{\prime}, z^{\prime}\right)=0$ is birational to a plane curve in the $x^{\prime} y^{\prime}$ plane $\mathcal{C}: R\left(x^{\prime}, y^{\prime}\right)=0$.

We will first illustrate how to approximate a parametric function $f(t)$ with piecewise quadratic function.

Algorithm 6.2 The input is a function $P(t), 0 \leq t \leq 1$ and an error bound $\delta$. The output is a rational quadratic function spline $B(t)$ such that the approximation error between $B(t)$ and $P(t)$ is less than $\delta$.

This is a classic approximation problem. We will not give the details.

We can now give the following algorithm to approximate the space curve $S C$ with rational quadratic space splines.

Algorithm 6.3 The inputs are a space curve $S C$ : Zero $(f, g)$ defined by $f(x, y, z)=0$ and $g(x, y, z)=0$ where $f(x, y, z), g(x, y, z) \in Z[x, y, z]$ are irreducible polynomials of degree larger than two, a bounding box $B=\left\{(x, y, z): x_{0} \leq x \leq x_{1}, y_{0} \leq y \leq y_{1}, z_{0} \leq z \leq z_{1}\right\}$, an error bound $\delta>0$. The outputs are several rational quadratic space spline curves $B_{i}(t), 1 \leq$ $i \leq r$, such that they give a $C^{1}$ approximation to $S C$ within the bounding box $B$ with the approximation error less than $\delta$.

1. Transform $S C: Z \operatorname{ero}(f, g)$ with a linear transformation $A$ such that it is birational to a plane curve $\mathcal{C}: R(x, y)=0$ with Theorem 6.1. Rewrite the resulted functions as $f, g, R$ and $\mathcal{C}$.

2. Assume that the birational map between $S C$ and $\mathcal{C}$ is defined as $x=x, y=y$ and $z=\frac{z_{0}(x, y)}{z_{1}(x, y)}$, where $z_{0}$ and $z_{1}$ are polynomials in $x$ and $y$.

3. Approximate $R=0$ with rational quadratic B-splines $\left(B_{x}(t), B_{y}(t)\right)$ according to the main algorithm described in section 5 .. We obtain $z(t)=\frac{z_{0}(x, y)}{z_{1}(x, y)}=\frac{z_{0}\left(B_{x}(t), B_{y}(t)\right)}{z_{1}\left(B_{x}(t), B_{y}(t)\right)}$.

4. Give a $\delta$-approximation $B_{z}(t)$ for $z=z(t)$ with Algorithm 6.2. Subdivide the space curve into segments according to the knots of $B_{z}(t)$.

5. Re-approximate each segment resulted from the step above and approximate it as steps 3 and 4 , ending with the quadratic approximation segments $\bar{B}(t)=\left(B_{x}(t), B_{y}(t), B_{z}(t)\right)$. We conclude that the approximation error is less than $\delta$.

6. Let $(x(t), y(t), z(t))=A^{-1}\left(B_{x}(t), B_{y}(t), B_{z}(t)\right)$ and $(x(t), y(t), z(t))$ is just what we need.

\subsection{Approximation to parametric spatial curves}

A similar algorithm can also be given to approximate a rational parametric space curve

$$
S C:(x, y, z)=(x(t), y(t), z(t)) .
$$

We first introduce the following definition of a proper curve. 
Definition 6.4 [9] (10) is called proper if, except a subset set of points on SC, for each $\left(x_{0}, y_{0}, z_{0}\right)$ on $S C$, there exists only one $t_{0}$ such that $x_{0}=x\left(t_{0}\right), y_{0}=y\left(t_{0}\right), z_{0}=z\left(t_{0}\right)$.

Theorem 6.5 [9] If (10) is not proper, we can find a new parameter $s=f(t) / g(t)$ where $f, g$ are polynomials in $t$ such that the re-parametrization of (10) in terms of $s x=x(s), y=$ $y(s), z=z(s)$ are proper.

Theorem 6.6 If (10) is proper, there exist an algorithm to give the following inversion map $t=r(x, y, z)$ where $r$ is a rational function in $x, y, z$.

With these results, we can approximate a rational parametric space curve with rational quadratic space splines.

Algorithm 6.7 The input is a spatial curve $S C:(x, y, z)=(x(t), y(t), z(t)), a \leq t \leq b$ and an error bound $\delta$. The outputs are rational quadratic splines such that they approximate $S C$ with the approximation error less than $\delta$.

1. Transform $S C$ into a proper form with Theorem 6.5 and rewrite them as $S C:(x, y, z)=$ $(x(t), y(t), z(t)), a \leq t \leq b$.

2. From $x=x(t), y=y(t)$, find the inversion map $t=t(x, y)$ with Theorem 6.6.

3. Approximate $x=x(t), y=y(t)$ with the methods proposed in [10]. Write the resulted spline as $B(s)=\left(B_{x}(s), B_{y}(s)\right)$.

4. Approximate $z(t)=z(t(x, y))=z\left(t\left(B_{x}(s), B_{y}(s)\right)\right)$ with Algorithm 6.2.

5. Subdivide each segment in $S C$ according to the segments in $z(s)$.

6. Re-approximate each segment as steps 3 and 4, ending with the quadratic approximation segments $\bar{B}(t)=\left(B_{x}(t), B_{y}(t), B_{z}(t)\right)$. We conclude that the approximation error is less than $\delta$ and $\bar{B}(t)$ is what we need.

\section{Conclusion}

In this paper, we try to give a simple and intuitive approximation of the plane algebraic curve with rational quadratic curves. The basic idea is to divide the curve into triangle convex segments which can be nicely approximated with quadratic Bézier curves and to connect the segments into certain maximal branches which can be globally approximated by quadratic B-splines. Some extension of the method to space curve is also illustrated. Experiments show that we can achieve high precision approximation with few segments. Instead of giving a power series for each approximated segment, the endpoint information and shoulder points are mainly used to express the segment. Since the geometric information is considered in the algorithm, the algorithm is easy to understand and many geometry characters of the approximated curve are kept. On the other hand, the algorithm involves the computation of the flexes of a plane curve, so it is especially efficient for a curve with no real inflection points. How to avoid the computation of flexes is an interesting work to do. It is also interesting to extend this algorithm to implicitly defined algebraic surfaces. 


\section{References}

[1] Abhyankar S.S. and Bajaj C., Automatic Parameterization of Rational Curves and Surfaces, III: Algebraic Plane Curves, Computer Aided Geometric Design, 5(4), 309-321, 1988.

[2] Bajaj C. and Xu G., Piecewise Approximations of Real Aglebraic Curves, J. Comp. Math., 15(1), 55-71, 1997.

[3] Blomgren R. and Fuhr R., Algorithm to Convert between Rational B-Spline and Rational Bézier Representation of Curves and Surfaces, Boeing Commericial Airplane Company, November 16, 1982.

[4] Bondy J. and Murty U., Graph Theory with Applications, the Macmillan Press Ltd., 1976.

[5] Chen F. and Wang W., Computing Real Inflection Points of Cubic Algebraic Curves, Computer Aided Geometric Design, 20(2), 101-117, 2003.

[6] Chuang J. and Hoffmann C., On Local Implicit Approximation and its Application, ACM Trans. on Graphics, 8(4), 298-324, 1989.

[7] Farin G., Curvature Continuity and Offsets for Piecewise Conics, ACM Trans. on Graphics, 8(2), 89-99, 1989.

[8] Gao X.S. and Chou S., On the Parameterization of Algebraic Curves, Applicable Algebra in Elementary Communication and Computing, 3, 27-38, 1992.

[9] Gao X.S. and Chou S., Implicitization of Rational Parametric Equations, Journal of Symbolic Computation, 14, 459-470, 1992.

[10] Gao X.S. and Li M., Approximate Implicitization of Plane Parametric Curves using Quadratic Splines. Submitted.

[11] Gonzalez-Vega L. and Necula I., Efficient Topology Determination of Implicitly Defined Algebraic Plane Curves, Computer Aided Geometric Design, 19(9), 719-743, 2002.

[12] Hong H., An Efficient Method for Analyzing the Topology of Plane Real Algebraic Curves, Mathematics and Computers in Simulation, 42(4-6), 571-582, 1996.

[13] Ihm I., and Naylor B., Piecewise Linear Approximations of Digitized Space Curves with Applications, Scientific Visualization of Physical Phenomena, ed. Patrikalakis N.M., Spring-Verlag, 545-569, 1991.

[14] Lee E., The Rational Bézier Representation for Conics, Geometric Modeling: Algorithm and New Trends, Farin G. (eds.), 3-19, SIAM, Philadelphia, 1985.

[15] Li Y. and Cripps R., Identification of Inflection Points and Cusps on Rational Curves, Computer Aided Geometric Design, 14(6), 491-497, 1997.

[16] Pottmann H., Leopoldseder S. and Hofer M., Approximation with Active B-spline Curves and Surfaces, Proc. Pacific Graphics 2002, Coquillart S., Shum H., Hu S. (eds), 8-25, IEEE Press, Los Alamitos, 2002.

[17] Safonova A. and Rossignac J., Compressed Piecewise-circular Approximations of 3D Curves, Computer Aided Design, 35(6), 533-547, 2003.

[18] Sederberg T. and Zheng J., Algebraic Methods for Computer Aided Geometric Design, in Handbook of Computer Aided Geometric Design, Farin G., Hoschek J. and Kim M.S. (eds), NorthHolland, Amsterdam, 2002.

[19] Sendra, J. and Winkler, F., Symbolic Parameterization Curves, J. of Symbolic Computation, 12, 607-632, 1991.

[20] Schicho, J., Rational Parameterization of Real Algebraic Surfaces. In Proc. ISSAC'98, 302-308. ACM Press, 1998.

[21] Walker R., Algebraic Curves, Springer Verlag, New York, 1978. 
[22] Wang W., Shape Reconstruction with Active Spline Curve, Int. Workshop on geom. Rep. and Proc. in Nano-Bio Scale, 15, Setp., Hangzhou, 2003. 